\title{
PPP-RTK functional models formulated with undifferenced and uncombined GNSS observations
}

\author{
Baocheng Zhang ${ }^{1,3^{*}}$, Pengyu Hou ${ }^{1,2}$, Jiuping Zha ${ }^{1}$ and Teng Liu ${ }^{1}$
}

\begin{abstract}
Technique PPP-RTK combines the advantages of both the Precise Point Positioning (PPP) and the Real-Time Kinematic (RTK) positioning. With the emergence of multi-frequency Global Navigation Satellite System (GNSS) observations, it is preferable to formulate PPP-RTK functional models based on original (undifferenced and uncombined) observations. While there exist many variants of the undifferenced and uncombined PPP-RTK models, a unified theoretical framework needs developing to link these variants. In this contribution, we formulate a class of undifferenced and uncombined PPP-RTK functional models in a systematic way and cast them in a unified framework. This framework classifies the models into a code-plus-phase category and a phase-only category. Each category covers a variety of measurement scenarios on the network side, ranging from small-, medium- to large-scale networks. For each scenario, special care has been taken of the distinct ionospheric constraints and the difference between Code Division Multiple Access (CDMA) and Frequency Division Multiple Access (FDMA) signals. The key to systematically formulating these models lies in how to deal with the rank deficiency problems encountered. We opt for the Singularity-basis (S-basis) theory, giving rise to the full-rank observation equations in which the estimable parameters turn out to be the functions of original parameters and those selected as the S-basis. In the sequel, it becomes straightforward to derive for each scenario the user model as it, more or less, amounts to the single-receiver network model. Benefiting from the presented theoretical framework, the relationships and differences between various undifferenced and uncombined PPP-RTK models become clear, which can lead to the better use of these models in a specific situation.
\end{abstract}

Keywords: Global navigation satellite system (GNSS), Ambiguity resolution-enabled precise point positioning (PPPRTK), Singularity-basis (S-basis), Undifferenced and uncombined, Frequency division multiple access (FDMA), Phaseonly

\section{Introduction}

Global Navigation Satellite Systems (GNSSs) have long been providing high-precision positioning services with two classical techniques: Precise Point Positioning (PPP) and Real-Time Kinematic (RTK) positioning (Leick et al., 2015; Teunissen \& Montenbruck, 2017). PPP utilizes precise satellite orbit and clock products to achieve

\footnotetext{
*Correspondence: b.zhang@whigg.ac.cn

1 State Key Laboratory of Geodesy and Earth's Dynamics, Innovation Academy for Precision Measurement Science and Technology, Chinese Academy of Sciences, Wuhan, China

Full list of author information is available at the end of the article
}

positioning accuracy at millimeter to centimeter level after a long convergence time (tens of minutes) (Kouba \& Héroux, 2001; Zumberge et al., 1997). While RTK provides accurate results in real time through ambiguity resolution, but it relies on (pseudo) observations provided by a nearby reference station or network (Odijk, 2002; Wübbena et al., 2001). To seek a promising technique, the ambiguity resolution-enabled precise point positioning, also known as PPP-RTK, emerged (Wübbena et al., 2005).

PPP-RTK takes the advantages of both PPP and RTK techniques. It extends the PPP by providing users, among 
others, the satellite phase biases, enabling single-receiver ambiguity resolution and achieving RTK-like positioning performance (Teunissen \& Khodabandeh, 2015). PPPRTK is more flexible than RTK (or network-RTK) since it inherits the State Space Representation (SSR) from PPP instead of the Observable Space Representation (OSR) adopted by RTK. Combining different SSR corrections estimated on the PPP-RTK network side produces various services: using satellite clock and orbit corrections makes PPP-RTK compatible with PPP (Gao \& Shen, 2002; Liu et al., 2017); adding satellite phase bias correction allows for PPP-AR (Geng et al., 2012; Zhang et al., 2019); and further including atmospheric corrections achieves rapid, high-precision PPP-RTK positioning ( $\mathrm{Li}$ \& Ge, 2011; Zha et al., 2021). Moreover, PPP-RTK can also be compatible with RTK by transforming SSR to OSR (Khodabandeh \& Teunissen, 2015).

Many PPP-RTK methods exist in the literature. Teunissen and Khodabandeh (2015) classified these methods into five types: distinct clock model (Teunissen et al., 2010), common clock model (Zhang et al., 2011), integer recover clock model (Laurichesse et al., 2009), decoupled satellite clock model (Collins et al., 2010), and Uncalibrated Phase Delay/Fractional Cycle Bias (UPD/ FCB) model (Ge et al., 2008). The first two adopt an undifferenced and uncombined formulation, while the last three choose the ionosphere-free combination at the start of the presentation and attempt to extend to the undifferenced and uncombined formulation (Gu et al., 2015; Naciri \& Bisnath, 2021). The undifferenced and uncombined formulation simplifies the stochastic model, preserves all parameters for possible constraints, and enables the process of the observations from any systems and frequencies, thus is simple, flexible, and attractive (Lannes \& Prieur, 2013; Odijk et al., 2016; Schönemann et al., 2011). Hence, we focus on the undifferenced and uncombined PPP-RTK in this contribution.

Teunissen et al. (2010) proposed for the first time the undifferenced and uncombined PPP-RTK with the choice of a distinct clock datum, while Zhang et al. (2011) suggested adopting the common clock datum. The common clock model makes receiver and satellite biases estimable instead of being absorbed into distinct clocks, bringing a stronger model if the biases are properly constrained. Researchers then extended the common clock undifferenced and uncombined PPP-RTK from dualfrequency GPS cases to multi-frequency and multi-GNSS situations (Khodabandeh \& Teunissen, 2016; Ma et al., 2020; Odijk et al., 2017; Psychas et al., 2021). Imposing different constraints on atmospheric delays yielded various PPP-RTK variants suitable for small-scale, mediumscale, and large-scale networks (Odijk et al., 2012; Wang et al., 2017; Zha et al., 2021; Zhang et al., 2018, 2019).
Even in a very special case where the network contains only one receiver, researchers also proposed the concept of single-station PPP-RTK (Khodabandeh, 2021). With the proposal of a new GLONASS ambiguity resolution method (Teunissen, 2019), the Frequency Division Multiple Access (FDMA) PPP-RTK became achievable (Zhang et al., 2021). To avoid the adverse effects of unmodeled code-related errors, one can exclude code observations and turn to phase-only PPP-RTK (Hou et al., 2022). While there exist many variants of the undifferenced and uncombined PPP-RTK models, but the discussions about their relationships and differences are limited. This motivates us to present these extended models in a systematic way and cast them in a unified framework.

In this contribution, we systematically present various undifferenced and uncombined PPP-RTK models formulated based on the code-plus-phase and phaseonly observations. Concerning the different scales of networks, we provide the ionosphere-float, ionosphereweighted, and ionosphere-fixed models. For each scenario, we consider both Code Division Multiple Access (CDMA) and FDMA systems. To address the rank deficiency problem underlying each model, we employ the Singularity-basis (S-basis) theory (Teunissen, 1985) to establish full-rank observation equations and elaborate estimable parameters. Moreover, we comprehensively compare the models in terms of estimable parameters, ambiguity resolution performances, and applicable situations.

The remainder of this paper proceeds as follows. Section 2 introduces how to apply the S-basis theory to identify and eliminate the rank deficiencies in GNSS observation equations. Section 3 presents the PPP-RTK models based on code-plus-phase observations, while Sect. 4 provides the models based on phase-only observations. Section 5 discusses and compares different models. Section 6 summarizes and concludes the whole study.

\section{Rank deficiency identification and elimination in GNSS observation equations}

Undifferenced and uncombined GNSS observation equations encounter a rank deficiency problem. This section introduces how to apply the S-basis theory to identify and eliminate rank deficiencies in GNSS observation equations.

\section{Undifferenced and uncombined GNSS observation equations}

Considering a network receiver $r(r=1, \ldots, n)$ that tracks a satellite $s(r=1, \ldots, m)$ at a frequency $j(r=1, \ldots, f)$, we write the undifferenced and uncombined GNSS code and phase observations as (Teunissen \& Montenbruck, 2017) 


$$
\begin{aligned}
\mathrm{E}\left[p_{r, j}^{s}\right]= & m_{r}^{s} \tau_{r}+\mathrm{d} t_{r} \\
& -\mathrm{d} t^{s}+\mu_{j} l_{r}^{s}+d_{r, j}^{s}-d_{, j}^{s} \\
\mathrm{E}\left[\phi_{r, j}^{s}\right]= & m_{r}^{s} \tau_{r}+\mathrm{d} t_{r}-\mathrm{d} t^{s} \\
& -\mu_{j} l_{r}^{s}+\delta_{r, j}^{s}-\delta_{, j}^{s}+\lambda_{j}^{s} z_{r, j}^{s}
\end{aligned}
$$

where E[.] denotes the expectation operator. $p_{r, j}^{s}$ and $\phi_{r, j}^{s}$ represent the observed-minus-computed code and phase observations, respectively, containing the receiver-to-satellite geometry and other corrections. The unknown parameters on the right side include: $\tau_{r}$ the dry component of the zenith tropospheric delay mapped to the slant direction by the function $m_{r}^{s}$; $\mathrm{d} t_{r}$ the receiver clock error; $\mathrm{d} t^{s}$ the satellite clock error; $l_{r}^{s}$ the slant ionospheric delay at the first frequency linked to other frequencies by $\mu_{j}=\left(\lambda_{j}^{s} / \lambda_{1}^{s}\right)^{2}$ with $\lambda_{j}^{s}$ the wavelength; $d_{r, j}^{s}$ the receiver code bias; $\delta_{r, j}^{s}$ the receiver phase bias; $d_{j}^{s}$ the satellite code bias; $\delta_{, j}^{s}$ the satellite phase bias; and $z_{r, j}^{s}$ the ambiguities in cycles scaled by the wavelength $\lambda_{j}^{s}$. Except for ambiguities, other parameters are expressed in length.

Equation (1) fits both CDMA and FDMA signals as the definitions of the receiver biases $\left(d_{r, j}^{s}\right.$ and $\left.\delta_{r, j}^{s}\right)$ and wavelength $\left(\lambda_{j}^{s}\right)$ contain a satellite index, implying the consideration of Inter-Frequency Bias (IFB) in GLONASS FDMA signals. For CDMA systems, the receiver biases and wavelength are reduced to satellite-independent quantities $\left(d_{r, j}, \delta_{r, j}\right.$, and $\left.\lambda_{j}\right)$.

\section{Rank deficiency identification and elimination}

Since the design matrix of Eq. (1) is rank-deficient, the observations lack the information to estimate all unknown parameters. Here, we introduce how to apply the S-basis theory (Teunissen, 1985) to identify and eliminate rank deficiencies. To keep this process simple but not lose generality, we take the rank deficiency between receiver and satellite clocks as an example. The design matrix of receiver and satellite clocks $\boldsymbol{A}$ reads

$$
\boldsymbol{A}=\left[\boldsymbol{e}_{f} \otimes \boldsymbol{I}_{n} \otimes \boldsymbol{e}_{m} \boldsymbol{e}_{f} \otimes\left(-\boldsymbol{e}_{n} \otimes \boldsymbol{I}_{m}\right)\right]
$$

where $\otimes$ denotes the Kronecker product, $\boldsymbol{e}_{(.)}$denotes the column vector of ones, and $\boldsymbol{I}_{(.)}$denotes the identity matrix. One can identify that the column-rank of matrix $\boldsymbol{A}$ equals $n+m-1$, implying the impossibility to estimate all $n+m$ receiver and satellite clock errors. Hence, we decompose the vector of the receiver and satellite clock errors $\mathrm{d} \boldsymbol{t}$ into an estimable vector $\mathrm{d} \hat{\boldsymbol{t}}$ and an inestimable part $\boldsymbol{V} \boldsymbol{\beta}$ (Odijk et al., 2016)

$$
\mathrm{d} \boldsymbol{t}=\mathrm{d} \hat{\boldsymbol{t}}+\boldsymbol{V} \boldsymbol{\beta}
$$

where $\boldsymbol{V}=\boldsymbol{e}_{n+m}$ is the basis matrix corresponding to the null space of matrix $A$, satisfying $A V=0$, while $\beta$ is the vector containing the minimum constraints on the parameters. The choice of $\boldsymbol{\beta}$ is not unique since $\mathrm{d} \boldsymbol{t}$ can be decomposed in many ways. Here, we select the clock error of the first receiver $\mathrm{d} t_{1}$ as the $\mathrm{S}$-basis and set $\boldsymbol{\beta}=\left[\mathrm{d} t_{1}\right]$, which is a one-dimensional vector. Substituting $\boldsymbol{\beta}$ into Eq. (3) yields

$$
\mathrm{d} \widehat{\boldsymbol{t}}=\mathrm{d} \boldsymbol{t}-\boldsymbol{V} \boldsymbol{\beta}=\left[\mathrm{d} \widehat{t}_{2}, \ldots, \mathrm{d} \widehat{t}_{n}, \mathrm{~d} \widehat{t}^{1}, \ldots, \mathrm{d} \widehat{t}^{m}\right]^{\mathrm{T}}
$$

where $\mathrm{d} \widehat{t}_{r}=\mathrm{d} t_{r}-\mathrm{d} t_{1}$ and $\mathrm{d} \widehat{t}^{s}=\mathrm{d} t^{s}-\mathrm{d} t_{1}$ are the estimable receiver and satellite clock errors, respectively. Substituting $\mathrm{d} \widehat{t}_{r}$ and $\mathrm{d} \widehat{t}^{s}$ into Eq. (1) yields

$$
\begin{aligned}
\mathrm{E}\left[p_{r, j}^{s}\right]= & m_{r}^{s} \tau_{r}+\mathrm{d} \widehat{t}_{r}-\mathrm{d} \widehat{t}^{s} \\
& +\mu_{j} l_{r}^{s}+d_{r, j}^{s}-d_{, j}^{s} \\
\mathrm{E}\left[\phi_{r, j}^{s}\right]= & m_{r}^{s} \tau_{r}+\mathrm{d} \widehat{t}_{r}-\mathrm{d} \widehat{t}^{s} \\
& -\mu_{j} l_{r}^{s}+\delta_{r, j}^{s}-\delta_{, j}^{s}+\lambda_{j}^{s} z_{r, j}^{s}
\end{aligned}
$$

where the rank deficiency between receiver and satellite clock errors is eliminated. For other rank deficiencies, one can adopt the same method to identify and eliminate them.

\section{Code-plus-phase PPP-RTK models}

This section works on both code and phase observations, forming the so-called code-plus-phase PPP-RTK models. Considering different constraints on the atmospheric delays, we formulate the ionosphere-float, ionosphereweighted, and ionosphere-fixed models. All models consider both CDMA and FDMA systems.

\section{lonosphere-float models}

In a larger-scale network, we parameterize the ionospheric delays without any constraints, forming the ionosphere-float models for both CDMA and FDMA systems.

\section{CDMA model}

Starting from CDMA observation equations in Eq. (1), we apply the S-basis theory to identify and eliminate the rank deficiencies in a step-by-step manner. To make this process straightforward, we introduce an identity in which a frequency-dependent quantity at the first two frequencies $(.)_{j=1,2}$ can be decomposed as (Teunissen \& Khodabandeh, 2015)

$$
(.)_{j=1,2}=(.)_{\mathrm{IF}}+\mu_{j}(.)_{, \mathrm{GF}}
$$


where $(.)_{, \mathrm{IF}}$ and $(.)_{\mathrm{GF}}$ are the ionosphere-free and geometry-free combinations, respectively, which are defined as.

$$
\begin{aligned}
(.)_{\mathrm{GF}} & =\frac{1}{\mu_{2}-\mu_{1}}\left[(.)_{, 2}-(.)_{1}\right] \\
(.)_{\mathrm{IF}} & =\frac{\mu_{2}}{\mu_{2}-\mu_{1}}(.)_{1}-\frac{\mu_{1}}{\mu_{2}-\mu_{1}}()_{, 2}
\end{aligned}
$$

Decomposing receiver and satellite code biases through Eq. (6), we rewrite Eq. (1) as

$$
\begin{aligned}
\mathrm{E}\left[p_{r, j}^{s}\right]= & m_{r}^{s} \tau_{r}+\mathrm{d} t_{r}-\mathrm{d} t^{s} \\
& +\mu_{j} l_{r}^{s}+\left(d_{r, \mathrm{IF}}+\mu_{j} d_{r, \mathrm{GF}}\right) \\
& -\left(d_{, \mathrm{IF}}^{s}+\mu_{j} d_{, \mathrm{GF}}^{s}\right)+\bar{d}_{r, j}-\bar{d}_{, j}^{s} \\
\mathrm{E}\left[\phi_{r, j}^{s}\right]= & m_{r}^{s} \tau_{r}+\mathrm{d} t_{r}-\mathrm{d} t^{s} \\
& -\mu_{j} l_{r}^{s}+\delta_{r, j}-\delta_{, j}^{s}+\lambda_{j} z_{r, j}^{s}
\end{aligned}
$$

with

$$
\begin{aligned}
& \bar{d}_{r, j}=d_{r, j}-d_{r, \mathrm{IF}}-\mu_{j} d_{r, \mathrm{GF}}(j>2) \\
& \bar{d}_{, j}^{s}=d_{, j}^{s}-d_{, \mathrm{IF}}^{\mathrm{S}}-\mu_{j} d_{, \mathrm{GF}}^{s}(j>2)
\end{aligned}
$$

being the recombined receiver and satellite biases. Of particulate note, although Eq. (9) holds for all frequencies and is equal to zero for $j=1,2$, we restrict them to $j>2$ for emphasizing that the recombined receiver and satellite biases exist only at the third frequency and above.

Four types of rank deficiencies then become clear: (1) between $\mathrm{d} t_{r}, d_{r, \mathrm{IF}}$, and $\delta_{r, j}$ of size $n$; (2) between $\mathrm{d} t_{r}, d_{\mathrm{IF}}^{s}$, and $\delta_{, j}^{s}$ of size $m$; (3) between $l_{r}^{s}, d_{r, \mathrm{GF}}$, and $\delta_{r, j}$ of size $n$; and (4) between $l_{r}^{s}, d_{, \mathrm{GF}}^{s}$, and $\delta_{j}^{s}$ of size $m$. We select $d_{r, \mathrm{IF}}$, $d_{\mathrm{IF}}^{s}, d_{r, \mathrm{GF}}$, and $d_{\mathrm{GF}}^{s}$ as the $\mathrm{S}$-basis to eliminate these rank deficiencies, yielding

$$
\begin{aligned}
\mathrm{E}\left[p_{r, j}^{s}\right]= & m_{r}^{s} \tau_{r}+\mathrm{d} \bar{t}_{r}-\mathrm{d} \bar{t}^{s} \\
& +\mu_{j} \bar{l}_{r}^{s}+\bar{d}_{r, j}-\bar{d}_{, j}^{s} \\
\mathrm{E}\left[\phi_{r, j}^{s}\right]= & m_{r}^{s} \tau_{r}+\mathrm{d} \bar{t}_{r}-\mathrm{d} \bar{t}^{s} \\
& -\mu_{j} \bar{l}_{r}^{s}+\bar{\delta}_{r, j}-\bar{\delta}_{, j}^{s}+\lambda_{j} z_{r, j}^{s}
\end{aligned}
$$

with

$$
\begin{aligned}
& \mathrm{d} \bar{t}_{r}=\mathrm{d} t_{r}+d_{r, \mathrm{IF}} \\
& \mathrm{d} \bar{t}^{s}=\mathrm{d} t^{s}+d_{, \mathrm{IF}}^{s} \\
& \bar{l}_{r}^{s}=l_{r}^{s}-d_{, \mathrm{GF}}^{s}+d_{r, \mathrm{GF}} \\
& \bar{\delta}_{r, j}=\delta_{r, j}-d_{r, \mathrm{IF}}+\mu_{j} d_{r, \mathrm{GF}} \\
& \bar{\delta}_{, j}^{s}=\delta_{, j}^{s}-d_{, \mathrm{IF}}^{s}+\mu_{j} d_{, \mathrm{GF}}^{s}
\end{aligned}
$$

being the recombined parameters. Except for the recombined ionospheric delay $\bar{l}_{r}^{s}$, other parameters are still inestimable.

We then consider two other types of rank deficiencies: (5) between $\mathrm{d} \bar{t}_{r}$ and $\mathrm{d} \bar{t}^{s}$ of size 1 ; and (6) between $\bar{d}_{r, j}$ and $\bar{d}_{, j}^{s}$ of size $f-2$. Selecting $\mathrm{d} \bar{t}_{1}$ and $\bar{d}_{1, j>2}$ as the $\mathrm{S}$-basis reforms the equations as

$$
\begin{aligned}
\mathrm{E}\left[p_{r, j}^{s}\right]= & m_{r}^{s} \tau_{r}+\mathrm{d} \tilde{t}_{r}-\mathrm{d} \tilde{t}^{s} \\
& +\mu_{j} \bar{l}_{r}^{s}+\tilde{d}_{r, j}-\tilde{d}_{, j}^{s} \\
\mathrm{E}\left[\phi_{r, j}^{s}\right]= & m_{r}^{s} \tau_{r}+\mathrm{d} \tilde{t}_{r}-\mathrm{d} \tilde{t}^{s} \\
& -\mu_{j} \bar{l}_{r}^{s}+\bar{\delta}_{r, j}-\bar{\delta}_{, j}^{s}+\lambda_{j} z_{r, j}^{s}
\end{aligned}
$$

with

$$
\begin{aligned}
\mathrm{d} \tilde{t}_{r} & =\mathrm{d} \bar{t}_{r}-\mathrm{d} \bar{t}_{1} \quad(r>1) \\
\mathrm{d} \tilde{t}^{s} & =\mathrm{d} \bar{t}^{s}-\mathrm{d} \bar{t}_{1} \\
\tilde{d}_{r, j} & =\bar{d}_{r, j}-\bar{d}_{1, j} \quad(r>1, j>2) \\
\tilde{d}_{, j}^{s} & =\bar{d}_{, j}^{s}-\bar{d}_{1, j} \quad(j>2)
\end{aligned}
$$

being the estimable receiver clock error, satellite clock error, receiver code bias, and satellite code bias, respectively.

We finally identify the remaining two types of rank deficiencies: (7) between $\bar{\delta}_{, j}^{s}$ and $z_{r, j}^{s}$ of size $f m$; and (8) between $\bar{\delta}_{r, j}$ and $z_{r, j}^{s}$ of size $f n$. Here, the choice of the $\mathrm{S}$-basis must keep the integer nature of the ambiguities. To this end, we select $z_{1, j}^{s}, z_{r \neq 1, j}^{1}+\bar{\delta}_{1, j}$ as the S-basis, yielding the full-rank model

$$
\begin{aligned}
\mathrm{E}\left[p_{r, j}^{s}\right]= & m_{r}^{s} \tau_{r}+\mathrm{d} \tilde{t}_{r}-\mathrm{d} \tilde{t}^{s} \\
& +\mu_{j} \bar{l}_{r}^{s}+\tilde{d}_{r, j}-\tilde{d}_{, j}^{s} \\
\mathrm{E}\left[\phi_{r, j}^{s}\right]= & m_{r}^{s} \tau_{r}+\mathrm{d} \tilde{t}_{r}-\mathrm{d} \tilde{t}^{s}-\mu_{j} \bar{l}_{r}^{s} \\
& +\tilde{\delta}_{r, j}-\tilde{\delta}_{, j}^{s}+\lambda_{j} \tilde{z}_{r, j}^{s}
\end{aligned}
$$

with

$$
\begin{aligned}
\tilde{\delta}_{r, j} & =\bar{\delta}_{r, j}-\bar{\delta}_{1, j}+\lambda_{j}\left(z_{r, j}^{1}-z_{1, j}^{1}\right) \quad(r>1) \\
\tilde{\delta}_{, j}^{s} & =\bar{\delta}_{, j}^{s}-\bar{\delta}_{1, j}-\lambda_{j} z_{1, j}^{s} \\
\tilde{z}_{r, j}^{s} & =\left(z_{r, j}^{s}-z_{1, j}^{s}\right)-\left(z_{r, j}^{1}-z_{1, j}^{1}\right) \quad(r>1, s>1)
\end{aligned}
$$

being the estimable receiver phase bias, satellite phase bias, and double-differenced ambiguities, respectively.

Equation (14) represents the full-rank model on the network side. Providing users with the satellite clock corrections, satellite biases, and optionally, the atmospheric 
delays, one can form the user model parameterizing integer ambiguities. For brevity, we do not correct the atmospheric delays but parameterize them as unknowns, thereby forming the full-rank user model as

$$
\begin{aligned}
\mathrm{E}\left[p_{u, j}^{s}+\mathrm{d} \tilde{t}^{s}+\tilde{d}_{, j}^{s}\right]= & \boldsymbol{e}_{u}^{s} \Delta \boldsymbol{x}+m_{u}^{s} \tau_{u} \\
& +\mathrm{d} \tilde{t}_{u}+\mu_{j} \bar{l}_{u}^{s}+\tilde{d}_{r, j} \\
\mathrm{E}\left[\phi_{u, j}^{s}+\mathrm{d} \tilde{t}^{s}+\tilde{\delta}_{, j}^{s}\right]= & \boldsymbol{e}_{u}^{s} \Delta \boldsymbol{x}+m_{u}^{s} \tau_{u} \\
& +\mathrm{d} \tilde{t}_{u}-\mu_{j} \bar{l}_{u}^{s}+\tilde{\delta}_{u, j}+\lambda_{j} \tilde{z}_{u, j}^{s}
\end{aligned}
$$

where $\Delta \boldsymbol{x}$ denotes the coordinate vector of a user receiver multiplied by the line-of-sight vector $\boldsymbol{e}_{u}^{s}$. The definitions of other parameters are consistent with those on the network side, replacing the network receiver index ' $r$ ' with a user receiver index ' $u$ '. Equation (16) (the user model) is equivalent to Eq. (14) (the network model) if Eq. (16) moves the satellite clock errors and satellite biases in Eq. (14) from the right side to the left side and parameterizes the user coordinates.

\section{FDMA model}

The FDMA model adopts the same S-basis as the CDMA model, thereby forming the equations as

$$
\begin{aligned}
\mathrm{E}\left[p_{r, j}^{s}\right]= & m_{r}^{s} \tau_{r}+\mathrm{d} \tilde{t}_{r}-\mathrm{d} \tilde{t}^{s} \\
& +\mu_{j} \bar{l}_{r}^{s}+\tilde{d}_{r, j}-\tilde{d}_{, j}^{s} \\
\mathrm{E}\left[\phi_{r, j}^{s}\right]= & m_{r}^{s} \tau_{r}+\mathrm{d} \tilde{t}_{r}-\mathrm{d} \tilde{t}^{s}-\mu_{j} \bar{l}_{r}^{s} \\
& +\tilde{\delta}_{r, j}-\tilde{\delta}_{, j}^{s}+\left(\lambda_{j}^{s} \bar{z}_{r, j}^{s}-\lambda_{j}^{1} \bar{z}_{r, j}^{1}\right)
\end{aligned}
$$

where $\bar{z}_{r, j}^{s}=z_{r, j}^{s}-z_{1, j}^{s}$ is the between-receiver single-differenced ambiguity. Since the estimable receiver biases $\left(\tilde{d}_{r>1, j}\right.$ and $\left.\tilde{\delta}_{r>1, j}\right)$ are the bias differences between two receivers, we ignore the between-receiver single-differenced IFB, making estimable receiver biases free of satellite identifiers. This is reasonable in the network where the receivers and the connected antennas and firmware are homogenous (Wanninger, 2012).

Ambiguities in Eq. (17) are still inestimable. Teunissen (2019) proposed to parameterize the ambiguities as

$$
\begin{aligned}
\mathrm{E}\left[p_{r, j}^{s}\right]= & m_{r}^{s} \tau_{r}+\mathrm{d} \tilde{t}_{r}-\mathrm{d} \tilde{t}^{s} \\
& +\mu_{j} \bar{l}_{r}^{s}+\tilde{d}_{r, j}-\tilde{d}_{, j}^{s} \\
\mathrm{E}\left[\phi_{r, j}^{s}\right]= & m_{r}^{s} \tau_{r}+\mathrm{d} \tilde{t}_{r}-\mathrm{d} \tilde{t}^{s} \\
& -\mu_{j} \bar{l}_{r}^{s}+\tilde{\delta}_{r, j}-\tilde{\delta}_{, j}^{s}+\frac{2848 \lambda_{j}^{0}}{a_{1} a_{s}} \tilde{\tilde{z}}_{r, j}^{s}
\end{aligned}
$$

where $\lambda_{i}^{0}$ denotes the wavelength of the GLONASS center frequency. $a_{s}=2848+\kappa^{s}$ with $\kappa^{s} \in[-7,+6]$ being the channel number of GLONASS satellites; $\tilde{\tilde{z}}_{r, j}^{s}=a_{1}\left(z_{r, j}^{s}-z_{1, j}^{s}\right)-a_{s}\left(z_{r, j}^{1}-z_{1, j}^{1}\right)$ is the estimable GLONASS ambiguity.

Although ambiguities in Eq. (18) are estimable and integers, the integer nature of the original ambiguities cannot be guaranteed. For rigorous ambiguity resolution, we must transform the estimable ambiguities to integer-estimable ones, whose definition is in Teunissen (2019).

Along a similar line with Eq. (16), we form the FDMA user model as

$$
\begin{aligned}
\mathrm{E}\left[p_{u, j}^{s}+\mathrm{d} \tilde{t}^{s}+\tilde{d}_{j, j}^{s}\right]= & \boldsymbol{e}_{u}^{s} \Delta \boldsymbol{x} \\
& +m_{u}^{s} \tau_{u}+\mathrm{d} \tilde{t}_{u}+\mu_{j} \bar{l}_{u}^{s}+\tilde{d}_{r, j} \\
\mathrm{E}\left[\phi_{u, j}^{s}+\mathrm{d} \tilde{t}^{s}+\tilde{\delta}_{, j}^{s}\right]= & \boldsymbol{e}_{u}^{s} \Delta \boldsymbol{x}+m_{u}^{s} \tau_{u} \\
& +\mathrm{d} \tilde{t}_{u}-\mu_{j} \bar{l}_{u}^{s}+\tilde{\delta}_{u, j}+\frac{2848 \lambda_{j}^{0}}{a_{1} a_{s}} \tilde{z}_{r, j}^{s}
\end{aligned}
$$

where the estimable ambiguities must be transformed to integer-estimable ambiguities.

\section{lonosphere-weighted models}

In medium-scale networks, we consider the spatial correlation between ionospheric delays by introducing a zeromean constraint to between-receiver single-differenced ionospheric delays

$$
\mathrm{E}\left[\hat{l}_{r}^{s}\right]=l_{r}^{s}-l_{1}^{s}
$$

where $\widehat{l}_{r}$ denotes the pseudo ionospheric observations set as zero. Its uncertainty is modeled by a stochastic model, for example, the stochastic model in Zha et al. (2021).

With the introduction of weighted ionospheric constraints, some rank deficiencies change. Additionally, one new rank deficiency between the satellite clock error and tropospheric delay occurs since tropospheric mapping functions are almost identical in medium-scale networks. This section clarifies these rank deficiencies and forms the ionosphere-weighted models for both CDMA and FDMA systems.

\section{CDMA model}

Combining pseudo ionospheric observations with GNSS code and phase observations, the size of the rank deficiency between $l_{r}^{s}, d_{r, \mathrm{GF}}$, and $\delta_{r, j}$ is no longer $n$, but only one. We select the geometry-free code bias of the first receiver $d_{1, \mathrm{GF}}$ 
as the S-basis instead of the code biases of all receivers $d_{r, \text { GF}}$. Keeping other S-basis unchanged, we form the ionospheric-weighted model as

$$
\begin{aligned}
\mathrm{E}\left[p_{r, j}^{s}\right]= & m_{r}^{s} \tau_{r}+\mathrm{d} \tilde{t}_{r}-\mathrm{d} \tilde{t}^{s}+\mu_{j} \tilde{l}_{r}^{s} \\
& +\mu_{j} \tilde{d}_{r, \mathrm{GF}}+\tilde{d}_{r, j}-\tilde{d}_{, j}^{s} \\
\mathrm{E}\left[\phi_{r, j}^{s}\right]= & m_{r}^{s} \tau_{r}+\mathrm{d} \tilde{t}_{r}-\mathrm{d} \tilde{t}^{s}-\mu_{j} \tilde{l}_{r}^{s} \\
& +\tilde{\tilde{\delta}}_{r, j}-\tilde{\delta}_{, j}^{s}+\lambda_{j} \tilde{z}_{r, j}^{s} \\
\mathrm{E}\left[\tilde{l}_{r}^{s}\right]= & \tilde{l}_{r}^{s}-\tilde{l}_{1}^{s}
\end{aligned}
$$

with

$$
\begin{aligned}
\tilde{l}_{r}^{s}= & l_{r}^{s}-d_{, \mathrm{GF}}^{s}+d_{1, \mathrm{GF}} \\
\tilde{d}_{r, \mathrm{GF}}= & d_{\mathrm{r}, \mathrm{GF}}-d_{1, \mathrm{GF}}(r>1) \\
\tilde{\tilde{\delta}}_{r, j}= & \delta_{r, j}-d_{r, \mathrm{IF}}-\delta_{1, j}+d_{1, \mathrm{IF}} \\
& +\lambda_{j}\left(z_{r, j}^{1}-z_{1, j}^{1}\right)(r>1)
\end{aligned}
$$

being the estimable ionospheric delay, receiver code bias, and receiver phase bias, respectively, which differ from the parameters of the ionosphere-float model. Of particular note, although the estimable ionospheric delays $\tilde{l}_{r}^{s}$ are not the original ionospheric delays $l_{r}^{s}$, constraints on the estimable ionospheric delays in Eq. (21) are equivalent to the constraints on the original ionospheric delays in Eq. (20), as we can verify that $\mathrm{E}\left[\tilde{l}_{r}^{s}\right]=\tilde{l}_{r}^{s}-\tilde{l}_{1}^{s}=l_{r}^{s}-l_{1}^{s}$

Since the tropospheric mapping functions are almost identical in a medium-scale network, an additional rank deficiency between the tropospheric delay and satellite clock error occurs. We select the tropospheric delay of the first receiver as the S-basis, yielding the full-rank ionosphere-weighted model

$$
\begin{aligned}
\mathrm{E}\left[p_{r, j}^{s}\right]= & m_{r}^{s} \tilde{\tau}_{r}+\mathrm{d} \tilde{t}_{r}-\mathrm{d} \tilde{\tilde{t}}^{s} \\
& +\mu_{j} \tilde{l}_{r}^{s}+\mu_{j} \tilde{d}_{r, \mathrm{GF}}+\tilde{d}_{r, j}-\tilde{d}_{, j}^{s} \\
\mathrm{E}\left[\phi_{r, j}^{s}\right]= & m_{r}^{s} \tilde{\tau}_{r}+\mathrm{d} \tilde{t}_{r}-\mathrm{d} \tilde{\tilde{t}}^{s} \\
& -\mu_{j} \tilde{l}_{r}^{s}+\tilde{\tilde{\delta}}_{r, j}-\tilde{\delta}_{, j}^{s}+\lambda_{j} \tilde{z}_{r, j}^{s} \\
\mathrm{E}\left[\hat{l}_{r}^{s}\right]= & \tilde{l}_{r}^{s}-\tilde{l}_{1}^{s}
\end{aligned}
$$

with

$$
\begin{aligned}
& \tilde{\tau}_{r}=\tau_{r}-\tau_{1}(r>1) \\
& \mathrm{d} \tilde{\tilde{t}}^{s}=\mathrm{d} \bar{t}^{s}-\mathrm{d} \bar{t}_{1}-m_{1}^{s} \tilde{\tau}_{1}
\end{aligned}
$$

being the estimable ionospheric delay and satellite clock error, respectively.

On the user side, we consider, among others, the atmospheric products, forming the full-rank user model as

$$
\begin{aligned}
\mathrm{E}\left[p_{u, j}^{s}+\mathrm{d} \tilde{\tilde{t}}^{s}+\tilde{d}_{, j}^{s}-m_{u}^{s} \tilde{\tau}_{u}\right]= & \boldsymbol{e}_{u}^{s} \Delta \boldsymbol{x} \\
& +\mathrm{d} \tilde{t}_{u}+\mu_{j} \tilde{l}_{u}^{s}+\mu_{j} \tilde{d}_{u, \mathrm{GF}}+\tilde{d}_{u, j} \\
\mathrm{E}\left[\phi_{u, j}^{s}+\mathrm{d} \tilde{\tilde{t}}^{s}+\tilde{\delta}_{, j}^{s}-m_{u}^{s} \tilde{\tau}_{u}\right]= & \boldsymbol{e}_{u}^{s} \Delta \boldsymbol{x} \\
& +\mathrm{d} \tilde{t}_{u}-\mu_{j} \tilde{l}_{u}^{s}+\tilde{\tilde{\delta}}_{u, j}+\lambda_{j} \tilde{z}_{u, j}^{s} \\
\mathrm{E}\left[\tilde{l}_{u}^{s}\right]= & \tilde{l}_{u}^{s}
\end{aligned}
$$

where $\tilde{\tau}_{u}$ and $\widehat{l}_{u}^{s}$ are the tropospheric and ionospheric delays, respectively, interpolated at the user station.

\section{FDMA model}

Along the similar line, one can form the FDMA model by replacing the CDMA estimable ambiguities with the FDMA ones. In this way, we write the FDMA network model as

$$
\begin{aligned}
\mathrm{E}\left[p_{r, j}^{s}\right]= & m_{r}^{s} \tilde{\tau}_{r}+\mathrm{d} \tilde{t}_{r}-\mathrm{d} \tilde{\tilde{t}}^{s}+\mu_{j} \tilde{l}_{r}^{s} \\
& +\mu_{j} \tilde{d}_{r, \mathrm{GF}}+\tilde{d}_{r, j}-\tilde{d}_{, j}^{s} \\
\mathrm{E}\left[\phi_{r, j}^{s}\right]= & m_{r}^{s} \tilde{\tau}_{r}+\mathrm{d} \tilde{t}_{r}-\mathrm{d} \tilde{\tilde{t}}^{s}-\mu_{j} \tilde{l}_{r}^{s} \\
& +\tilde{\tilde{\delta}}_{r, j}-\tilde{\delta}_{, j}^{s}+\frac{2848 \lambda_{j}^{0}}{a_{1} a_{s}} \tilde{\tilde{z}}_{r, j}^{s} \\
\mathrm{E}\left[\tilde{l}_{r}^{s}\right]= & \tilde{l}_{r}^{s}-\tilde{l}_{1}^{s}
\end{aligned}
$$

while the user mode reads

$$
\begin{aligned}
& \mathrm{E}\left[p_{u, j}^{s}+\mathrm{d} \tilde{\tilde{t}}^{s}+\tilde{d}_{, j}^{s}-m_{u}^{s} \tilde{\tau}_{u}\right]=\boldsymbol{e}_{u}^{s} \Delta \boldsymbol{x}+\mathrm{d} \tilde{t}_{u} \\
& \quad+\mu_{j} \tilde{l}_{u}^{s}+\mu_{j} \tilde{d}_{u, \mathrm{GF}}+\tilde{d}_{u, j} \\
& \mathrm{E}\left[\phi_{u, j}^{s}+\mathrm{d} \tilde{\tilde{t}}^{s}+\tilde{\delta}_{, j}^{s}-m_{u}^{s} \tilde{\tau}_{u}\right]=\boldsymbol{e}_{u}^{s} \Delta \boldsymbol{x}+\mathrm{d} \tilde{t}_{u} \\
& \quad-\mu_{j} \tilde{l}_{u}^{s}+\tilde{\tilde{\delta}}_{u, j}+\frac{2848 \lambda_{j}^{0}}{a_{1} a_{s}} \tilde{\tilde{z}}_{u, j}^{s} \\
& \mathrm{E}\left[\tilde{l}_{u}^{s}\right]=\tilde{l}_{u}^{s}
\end{aligned}
$$

where the estimable ambiguities must be transformed to integer-estimable ambiguities for rigorous ambiguity resolution.

\section{lonosphere-fixed models}

In small-scale networks, we assume that atmospheric delays at all stations are identical. Based on this assumption, we investigate how the rank deficiencies change 
and form the full-rank ionosphere-fixed models for both CDMA and FDMA systems.

\section{CDMA model}

Assuming that $m_{1}^{s} \tau_{1}=\cdots=m_{n}^{s} \tau_{n}=\tau^{s} \quad$ and $l_{1}^{s}=\ldots l_{n}^{s}=l^{s}$, the tropospheric delay $\tau^{s}$ can be directly absorbed into satellite clock errors, while the rank deficiencies related to the ionospheric delays are the same as those in the ionosphere-weighted model. We follow the S-basis selected for the ionosphere-weighted model and write the ionosphere-fixed model as

$$
\begin{aligned}
& \mathrm{E}\left[p_{r, j}^{s}\right]=\mathrm{d} \tilde{t}_{r}-\mathrm{d} \overline{\bar{t}}+\mu_{j} \bar{l}+\mu_{j} \tilde{d}_{r, \mathrm{GF}}+\tilde{d}_{r, j}-\tilde{d}_{, j}^{s} \\
& \mathrm{E}\left[\phi_{r, j}^{s}\right]=\mathrm{d} \tilde{t}_{r}-\mathrm{d} \bar{t}-\mu_{j} \bar{l}+\tilde{\tilde{\delta}}_{r, j}-\tilde{\delta}_{, j}^{s}+\lambda_{j} \tilde{z}_{r, j}^{s}
\end{aligned}
$$

with

$$
\begin{aligned}
& \mathrm{d} \overline{\bar{t}}=\mathrm{d} t^{s}+d_{, \mathrm{IF}}^{s}-\tau^{s}-\mathrm{d} t_{1}-d_{1, \mathrm{IF}} \\
& \overline{\bar{l}}=l^{s}-d_{, \mathrm{GF}}^{s}+d_{1, \mathrm{GF}}
\end{aligned}
$$

being the estimable satellite clock error and ionospheric delay, respectively.

On the user side, we directly use the ionospheric delay estimated on the network side to correct the ionospheric delay at a user station. Along with other corrections, we form the user model as

$$
\begin{aligned}
& \mathrm{E}\left[p_{r, j}^{s}+\mathrm{d} \overline{\bar{t}}-\mu_{j} \overline{\bar{l}}+\tilde{d}_{, j}^{s}\right]=\boldsymbol{e}_{u}^{s} \Delta \boldsymbol{x}+\mathrm{d} \tilde{t}_{r}+\mu_{j} \tilde{d}_{r, \mathrm{GF}}+\tilde{d}_{r, j} \\
& \mathrm{E}\left[\phi_{r, j}^{s}+\mathrm{d} \overline{\bar{t}}^{s}+\mu_{j} \overline{\bar{l}}+\tilde{\delta}_{, j}^{s}\right]=\boldsymbol{e}_{u}^{s} \Delta \boldsymbol{x}+\mathrm{d} \tilde{t}_{r}+\tilde{\tilde{\delta}}_{r, j}+\lambda_{j} \tilde{z}_{r, j}^{s}
\end{aligned}
$$

where the tropospheric delays are actually corrected since they are absorbed in the satellite clock errors.

\section{FDMA model}

It is now straightforward to extend the CDMA models to the FDMA models: the network model

$$
\begin{aligned}
\mathrm{E}\left[p_{r, j}^{s}\right]= & \mathrm{d} \tilde{t}_{r}-\mathrm{d} \overline{\bar{t}}+\mu_{j} \overline{\bar{l}} \\
& +\mu_{j} \tilde{d}_{r, \mathrm{GF}}+\tilde{d}_{r, j}-\tilde{d}_{, j}^{s} \\
\mathrm{E}\left[\phi_{r, j}^{s}\right]= & \mathrm{d} \tilde{t}_{r}-\mathrm{d} \overline{\bar{t}}-\mu_{j} \overline{\bar{l}} \\
& +\tilde{\tilde{\delta}}_{r, j}-\tilde{\delta}_{, j}^{s}+\frac{2848 \lambda_{j}^{0}}{a_{1} a_{s}} \tilde{z}_{r, j}^{s}
\end{aligned}
$$

$$
\begin{gathered}
\mathrm{E}\left[p_{r, j}^{s}+\mathrm{d} \overline{\bar{t}}-\mu_{j} \bar{l}+\tilde{d}_{, j}^{s}\right]=\boldsymbol{e}_{u}^{s} \Delta \boldsymbol{x} \\
+\mathrm{d} \tilde{t}_{r}+\mu_{j} \tilde{d}_{r, \mathrm{GF}}+\tilde{d}_{r, j} \\
\mathrm{E}\left[\phi_{r, j}^{s}+\mathrm{d} \overline{\bar{t}}+\mu_{j} \overline{\bar{l}}+\tilde{\delta}_{, j}^{s}\right]=\boldsymbol{e}_{u}^{s} \Delta \boldsymbol{x} \\
\quad+\mathrm{d} \tilde{t}_{r}+\tilde{\tilde{\delta}}_{r, j}+\frac{2848 \lambda_{j}^{0}}{a_{1} a_{s}} \tilde{\tilde{z}}_{u, j}^{s}
\end{gathered}
$$

where we emphasize again the necessity to transform the estimable ambiguities to integer-estimable ambiguities.

\section{Phase-only PPP-RTK models}

Code-plus-phase model performs well in the case the code biases are properly modeled. However, some code biases are not only receiver-related but also satelliterelated, for example, the code IFB in GLONASS observations and even some in CDMA observations, typically the multipath. These biases are difficult to be modeled and could degrade the positioning performance. To avoid the adverse effects of these unmodeled code errors, we exclude the code observations and only use the phase observations to realize PPP-RTK. Due to the exclusion of code observations, phase-only observation equations encounter additional rank deficiencies. This section further eliminates these rank deficiencies and forms the fullrank phase-only PPP-RTK models.

\section{lonosphere-float models}

We also start with the ionosphere-float model, which parameterizes ionospheric delays without any constraints.

\section{CDMA model}

Starting from phase observation equations in Eq. (14), we identify the additional rank deficiencies caused by the exclusion of code observations. To make it straightforward, we decompose the receiver and satellite phase biases through Eq. (6), and rewrite the phase observations as

$$
\begin{aligned}
\mathrm{E}\left[\phi_{r, j}^{s}\right]= & m_{r}^{s} \tau_{r}+\mathrm{d} \tilde{t}_{r}-\mathrm{d} \tilde{t}^{s}-\mu_{j} \bar{l}_{r}^{s} \\
& +\tilde{\delta}_{r, \mathrm{IF}}+\mu_{j} \tilde{\delta}_{r, \mathrm{GF}}-\tilde{\delta}_{, \mathrm{IF}}^{s} \\
& -\mu_{j} \tilde{\delta}_{, \mathrm{GF}}^{s}+\hat{\delta}_{r, j}-\hat{\delta}_{, j}^{s}+\lambda_{j} \tilde{z}_{r, j}^{s}
\end{aligned}
$$

with

and the user model 


$$
\begin{aligned}
& \hat{\delta}_{r, j}=\tilde{\delta}_{r, j}-\tilde{\delta}_{r, \mathrm{IF}}-\mu_{j} \tilde{\delta}_{r, \mathrm{GF}}(r>1, j>2) \\
& \hat{\delta}_{, j}^{s}=\tilde{\delta}_{, j}^{s}-\tilde{\delta}_{, \mathrm{IF}}^{s}-\mu_{j} \tilde{\delta}_{, \mathrm{GF}}^{s}(j>2)
\end{aligned}
$$

the estimable receiver and satellite phase biases at the third frequency and above.

Similar to the rank deficiencies related to the receiver code biases in Eq. (8), Eq. (33) encounters four types of rank deficiencies related to the receiver phase biases: (1) between $\mathrm{d} \tilde{t}_{r}$ and $\tilde{\delta}_{r, \text { IF }}$ of size $n$; (2) between $\mathrm{d} \tilde{t}^{s}$ and $\tilde{\delta}_{\text {,IF }}^{s}$ of size $m$; (3) between $\bar{l}_{r}^{s}$ and $\tilde{\delta}_{r, \mathrm{GF}}$ of size $n$; and (4) between $\bar{l}_{r}^{s}$ and $\tilde{\delta}_{, \mathrm{GF}}^{s}$ of size $m$. We select $\tilde{\delta}_{r, \mathrm{IF}}, \tilde{\delta}_{\text {,IF }}^{s}, \tilde{\delta}_{r, \mathrm{GF}}$, and $\tilde{\delta}_{, \mathrm{GF}}^{s}$ as the $\mathrm{S}$-basis, forming the equations as

$$
\begin{aligned}
\mathrm{E}\left[\phi_{r, j}^{s}\right]= & m_{r}^{s} \tau_{r}+\mathrm{d} \hat{t}_{r}-\mathrm{d} \hat{t}^{s}-\mu_{j} \hat{l}_{r}^{s} \\
& +\hat{\delta}_{r, j}+\hat{\delta}_{, j}^{s}+\lambda_{j} \tilde{z}_{r, j}^{s}
\end{aligned}
$$

with

$$
\begin{aligned}
& \mathrm{d} \hat{t}_{r}=\mathrm{d} \tilde{t}_{r}+\tilde{\delta}_{r, \mathrm{IF}}(r>1) \\
& \mathrm{d} \hat{t}^{s}=\mathrm{d} \tilde{t}^{s}+\tilde{\delta}_{, \mathrm{IF}}^{s} \\
& \hat{l}_{r}^{s}=\bar{l}_{r}^{s}-\tilde{\delta}_{r, \mathrm{GF}}+\tilde{\delta}_{, \mathrm{GF}}^{s}
\end{aligned}
$$

being the redefined receiver clock error, satellite clock error, and ionospheric delay, respectively.

Equation (35) still has one additional rank deficiency: 5) between $\hat{l}_{r}^{s}$ and $\tilde{z}_{r, j}^{s}$ of size $(n-1)(m-1)$. We select the ambiguities at the first frequency $\tilde{z}_{r, j=1}^{s}$ as the $\mathrm{S}$-basis, forming the full-rank model as

$$
\begin{aligned}
\mathrm{E}\left[\phi_{r, j}^{s}\right]= & m_{r}^{s} \tau_{r}+\mathrm{d} \hat{t}_{r}-\mathrm{d} \hat{t}^{s}-\mu_{j} \hat{\hat{l}}_{r}^{s} \\
& +\hat{\delta}_{r, j}+\hat{\delta}_{, j}^{s}+\lambda_{j} \hat{\tilde{z}}_{r, j}^{s}
\end{aligned}
$$

with

$$
\begin{aligned}
& \hat{\hat{l}}_{r}^{s}=\hat{l}_{r}^{s}-\lambda_{1} \tilde{z}_{r, 1}^{s} \\
& \hat{\tilde{z}}_{r, j}^{s}=\tilde{z}_{r, j}^{s}-\frac{\lambda_{j}}{\lambda_{1}} \tilde{z}_{r, 1}^{s}(j>1)
\end{aligned}
$$

being the estimable ionospheric delay and ambiguities, respectively.

To keep the integer nature of ambiguities, we define the simplest integer ratio $\frac{w_{2, j}}{w_{1, j}}$ that satisfies $\frac{w_{2, j}}{w_{1, j}}=\frac{\lambda_{j}}{\lambda_{1}}$, where $w_{1, j}$ and $w_{2, j}$ are integers. Replacing $\frac{\lambda_{j}}{\lambda_{1}}$ with $\frac{w_{2, j}}{w_{1, j}}$ yields

$$
\begin{aligned}
\mathrm{E}\left[\phi_{r, j}^{s}\right]= & m_{r}^{s} \tau_{r}+\mathrm{d} \hat{t}_{r}-\mathrm{d} \hat{t}^{s}-\mu_{j} \hat{\jmath}_{r}^{s} \\
& +\hat{\delta}_{r, j}+\hat{\delta}_{, j}^{s}+\frac{\lambda_{j}}{w_{1, j}} \hat{z}_{r, j}^{s}
\end{aligned}
$$

with

$$
\hat{\hat{z}}_{r, j}^{s}=w_{1, j} \tilde{z}_{r, j}^{s}-w_{2, j} \tilde{z}_{r, 1}^{s}(j>1)
$$

the integer-estimable ambiguities, to which a rigorous ambiguity resolution is applicable.

Users receive the satellite clock errors and satellite phase biases to form the full-rank model as

$$
\begin{gathered}
\mathrm{E}\left[\phi_{u, j}^{s}+\mathrm{d} \hat{t}^{s}+\hat{\delta}_{, j}^{s}\right]=\boldsymbol{e}_{u}^{s} \Delta \boldsymbol{x}+m_{u}^{s} \tau_{u} \\
+\mathrm{d} \hat{t}_{u}-\mu_{j} \hat{\hat{l}}_{u}^{s}+\hat{\delta}_{u, j}+\frac{\lambda_{j}}{w_{1, j}} \hat{\hat{z}}_{u, j}^{s}
\end{gathered}
$$

where the satellite phase biases are provided to the observations at the third frequency and above.

\section{FDMA model}

Again, replacing $\frac{\lambda_{j}}{w_{1, j}} \hat{\vec{z}}_{r, j}^{s}$ with $\frac{2848 \lambda_{j}^{0}}{w_{1, j} a_{1} a_{s}} \bar{z}_{r, j}^{s}$ forms the estimable network FDMA model

$$
\begin{aligned}
\mathrm{E}\left[\phi_{r, j}^{s}\right]= & m_{r}^{s} \tau_{r}+\mathrm{d} \hat{t}_{r}-\mathrm{d} \hat{t}^{s}-\mu_{j} \hat{\hat{l}}_{r}^{s} \\
& +\hat{\delta}_{r, j}+\hat{\delta}_{, j}^{s}+\frac{2848 \lambda_{j}^{0}}{w_{1, j} a_{1} a_{s}} \breve{z}_{r, j}^{s}
\end{aligned}
$$

and the estimable user FDMA model

$$
\begin{gathered}
\mathrm{E}\left[\phi_{u, j}^{s}+\mathrm{d} \hat{t}^{s}+\hat{\delta}_{, j}^{s}\right]=\boldsymbol{e}_{u}^{s} \Delta \boldsymbol{x}+m_{u}^{s} \tau_{u} \\
+\mathrm{d} \hat{t}_{u}-\mu_{j} \hat{\hat{l}}_{u}^{s}+\hat{\delta}_{u, j}+\frac{2848 \lambda_{j}^{0}}{w_{1, j} a_{1} a_{s}} \widetilde{z}_{u, j}^{s}
\end{gathered}
$$

with

$$
\breve{z}_{r, j}^{s}=w_{1, j} \tilde{z}_{r, j}^{s}-w_{2, j} \tilde{z}_{r, 1}^{s}(j>1)
$$

the estimable FDMA ambiguities which must be transformed to integer-estimable ambiguities for ambiguity resolution.

\section{lonosphere-weighted models}

Combining pseudo ionospheric observations with phase observations, this section reidentifies the rank deficiencies and forms the phase-only ionosphere-weighted models for both CDMA and FDMA systems.

\section{CDMA model}

With the introduction of pseudo ionospheric observations, the size of the rank deficiency between $\bar{l}_{r}^{s}$ and $\tilde{\delta}_{r, \mathrm{GF}}$ is no longer $n$, but only one. We select $\tilde{\delta}_{1, \mathrm{GF}}$, instead of $\tilde{\delta}_{r, \mathrm{GF}}$, as the S-basis to eliminate this rank deficiency, while the other $\mathrm{S}$-basis related to receiver biases are consistent with those in the ionosphere-float model. Moreover, we select the 
tropospheric delay of the first receiver to eliminate the rank deficiency between the tropospheric delay and satellite clock error. In this way, we write the CDMA ionosphereweighted network model as

$$
\begin{aligned}
\mathrm{E}\left[\phi_{r, j}^{s}\right]= & m_{r}^{s} \tilde{\tau}_{r}+\mathrm{d} \hat{t}_{r}-\mathrm{d} \tilde{\hat{t}}^{s}-\mu_{j} \tilde{\hat{l}}_{r}^{s} \\
& +\mu_{j} \tilde{\hat{\delta}}_{r, \mathrm{GF}}+\hat{\delta}_{r, j}+\hat{\delta}_{, j}^{s}+\lambda_{j} \tilde{z}_{r, j}^{s} \\
\mathrm{E}\left[\tilde{l}_{r}^{s}\right]= & \tilde{\hat{l}}_{r}^{s}-\tilde{\hat{l}}_{1}^{s}
\end{aligned}
$$

with

$$
\begin{aligned}
& \mathrm{d} \hat{\hat{t}}^{s}=\mathrm{d} \tilde{\tilde{t}}^{s}+\tilde{\delta}_{, \mathrm{IF}}^{s} \\
& \tilde{\hat{l}}_{r}^{s}=\tilde{l}_{r}^{s}-\tilde{\tilde{\delta}}_{1, \mathrm{GF}}+\tilde{\delta}_{, \mathrm{GF}}^{s} \\
& \tilde{\hat{\delta}}_{r, \mathrm{GF}}=\tilde{\tilde{\delta}}_{r, \mathrm{GF}}-\tilde{\tilde{\delta}}_{1, \mathrm{GF}}(r>1)
\end{aligned}
$$

being the estimable satellite clock error, ionospheric delay, and between-receiver differential phase bias, respectively. Note that, Eq. (45) is already estimable since the rank deficiency between the ionospheric delays and ambiguities no longer exists due to the introduction of ionospheric constraints.

We write the CDMA ionospheric-weighted user model as

$$
\begin{aligned}
& \mathrm{E}\left[\phi_{u, j}^{s}+\mathrm{d} \tilde{\hat{t}}^{s}+\hat{\delta}_{, j}^{s}-m_{u}^{s} \tilde{\tau}_{u}\right]=\boldsymbol{e}_{u}^{s} \Delta \boldsymbol{x} \\
& \quad+\mathrm{d} \hat{t}_{u}-\mu_{j} \tilde{\hat{l}}_{u}^{s}+\mu_{j} \tilde{\hat{\delta}}_{u, \mathrm{GF}}+\hat{\delta}_{u, j}+\lambda_{j} \tilde{z}_{u, j}^{s} \\
& \mathrm{E}\left[\tilde{l}_{u}^{s}\right]=\tilde{\hat{l}}_{u}^{s}
\end{aligned}
$$

where we emphasize again that satellite phase biases are provided only to observations at the third frequency and above.

\section{FDMA model}

Similarly, we form the FDMA network model as

$$
\begin{aligned}
\mathrm{E}\left[\phi_{r, j}^{s}\right]= & m_{r}^{s} \tilde{\tau}_{r}+\mathrm{d} \hat{t}_{r}-\mathrm{d} \tilde{\hat{t}}^{s}-\mu_{j} \tilde{\hat{l}}_{r}^{s} \\
& +\mu_{j} \tilde{\hat{\delta}}_{r, \mathrm{GF}}+\hat{\delta}_{r, j}+\hat{\delta}_{, j}^{s}+\frac{2848 \lambda_{j}^{0}}{a_{1} a_{s}} \tilde{\tilde{z}}_{r, j}^{s} \\
\mathrm{E}\left[\hat{l}_{r}^{s}\right]= & \tilde{\hat{l}}_{r}^{s}-\tilde{\hat{l}}_{1}^{s}
\end{aligned}
$$

and the user model as

$$
\begin{aligned}
& \mathrm{E}\left[\phi_{u, j}^{s}+\mathrm{d} \tilde{\hat{t}}^{s}+\hat{\delta}_{, j}^{s}-m_{u}^{s} \tilde{\tau}_{u}\right]=\boldsymbol{e}_{u}^{s} \Delta \boldsymbol{x}+\mathrm{d} \hat{t}_{u} \\
& -\mu_{j} \tilde{\hat{l}}_{u}^{s}+\mu_{j} \tilde{\hat{\delta}}_{u, \mathrm{GF}}+\hat{\delta}_{u, j}+\frac{2848 \lambda_{j}^{0}}{a_{1} a_{s}} \tilde{\tilde{z}}_{r, j}^{s} \\
& \mathrm{E}\left[\tilde{l}_{u}^{s}\right]=\tilde{\hat{l}}_{u}^{s}
\end{aligned}
$$

where the estimable ambiguities are the same as those in the code-plus-phase model.

\section{lonosphere-fixed models}

Based on the assumptions that $m_{1}^{s} \tau_{1}=\cdots=m_{n}^{s} \tau_{n}=\tau^{s}$ and $l_{1}^{s}=\ldots l_{n}^{s}=l^{s}$, this section provides the ionospherefixed models for both CDMA and FDMA systems.

\section{CDMA model}

From the experiences in the derivation of code-plus-phase models, we first lump the tropospheric delay with the satellite clock error, then adopt the same S-basis in the phaseonly ionosphere-weighted model, and finally, form the ionosphere-fixed network model as

$$
\begin{aligned}
\mathrm{E}\left[\phi_{r, j}^{s}\right]= & \mathrm{d} \hat{t}_{r}-\mathrm{d} \hat{\hat{t}}^{s}-\mu_{j} \tilde{\hat{l}}^{s}+\mu_{j} \tilde{\hat{\delta}}_{r, \mathrm{GF}} \\
& +\hat{\delta}_{r, j}+\hat{\delta}_{, j}^{s}+\lambda_{j} \tilde{z}_{r, j}^{s}
\end{aligned}
$$

with

$$
\begin{aligned}
\hat{\mathrm{d}} \hat{t}^{s} & =\mathrm{d} \overline{\bar{t}}+\tilde{\delta}_{, \mathrm{IF}}^{s} \\
\tilde{\hat{l}}^{s} & =\overline{\bar{l}}^{s}-\tilde{\tilde{\delta}}_{1, \mathrm{GF}}+\tilde{\delta}_{, \mathrm{GF}}^{s}
\end{aligned}
$$

the estimable satellite clock and ionospheric delay, respectively.

The user model reads

$$
\begin{gathered}
\mathrm{E}\left[\phi_{u, j}^{s}+\mathrm{d} \hat{\hat{t}}^{s}+\mu_{j} \tilde{\hat{l}}^{s}+\hat{\delta}_{, j}^{s}\right]=\boldsymbol{e}_{u}^{s} \Delta \boldsymbol{x} \\
+\mathrm{d} \hat{t}_{u}+\mu_{j} \tilde{\hat{\delta}}_{u, \mathrm{GF}}+\hat{\delta}_{u, j}+\lambda_{j} \tilde{z}_{u, j}^{s}
\end{gathered}
$$

where the satellite clock errors, ionospheric delays, and satellite phase biases are corrected.

\section{FDMA model}

Finally, we present the FDMA network model as

$$
\begin{aligned}
\mathrm{E}\left[\phi_{r, j}^{s}\right]= & \mathrm{d} \hat{t}_{r}-\mathrm{d} \hat{\hat{t}}^{s}-\mu_{j} \tilde{\hat{l}}^{s}+\mu_{j} \tilde{\hat{\delta}}_{r, \mathrm{GF}} \\
& +\hat{\delta}_{r, j}+\hat{\delta}_{, j}^{s}+\frac{2848 \lambda_{j}^{0}}{a_{1} a_{s}} \tilde{z}_{r, j}^{s}
\end{aligned}
$$


and the user model as

$$
\begin{aligned}
& \mathrm{E}\left[\phi_{u, j}^{s}+\mathrm{d} \hat{\hat{t}}^{s}+\mu_{j} \tilde{\hat{l}}^{s}+\hat{\delta}_{, j}^{s}\right] \\
& =\boldsymbol{e}_{u}^{s} \Delta \boldsymbol{x}+\mathrm{d} \hat{t}_{u}+\mu_{j} \tilde{\hat{\delta}}_{u, \mathrm{GF}}+\hat{\delta}_{u, j}+\frac{2848 \lambda_{j}^{0}}{a_{1} a_{s}} \tilde{\tilde{z}}_{r, j}^{s}
\end{aligned}
$$

where only the CDMA ambiguities are replaced with FDMA ambiguities.

\section{Discussions}

This section discusses the differences between the CDMA and FDMA models, compares the estimable parameters of the ionosphere-float, ionosphere-weighted, and ionosphere-fixed models, and clarifies the relationship between the code-plus-phase and phase-only models.

\section{CDMA vs. FDMA}

FDMA PPP-RTK models adopt the same S-basis selected by the CDMA PPP-RTK models, forming the between-receiver single-differenced receiver biases, e.g., Eqs. (13) and (15). Taking advantage of this formulation, FDMA PPP-RTK avoids the effects of IFB in the sense that between-receiver singledifferenced code IFB is ignorable in a network employing homogeneous receivers, while the phase counterpart is negligible even in heterogeneous networks (Wanninger, 2012). As a consequence, the FDMA and CDMA PPP-RTK models contain the same estimable parameters, except for ambiguities, e.g., Eqs. (14) and (18). We further discuss the CDMA and FDMA estimable ambiguities below.

The key to PPP-RTK is ambiguity resolution. Teunissen and Khodabandeh (2015) indicated that PPP-RTK fixes double-differenced ambiguities on both the network and user sides, regardless the methods adopted. Here, we further clarify that PPP-RTK fixes a linear combination of the ambiguities, typically, the double-differenced ambiguities. It is straightforward for CDMA systems to form doubledifferenced ambiguities, while this formulation fails for the FDMA system since it adopts different frequencies to identify satellites. To ensure the estimability of the FDMA PPPRTK model, we adopt a specific linear combination of FDMA ambiguities proposed in Teunissen (2019). Here, we write again the double-differenced CDMA ambiguities and the estimable FDMA ambiguities along with their coefficients

$$
\begin{aligned}
& \text { CDMA }: \lambda_{j} \tilde{z}_{r, j}^{s}=\lambda_{j}\left(z_{r, j}^{s}-z_{1, j}^{s}-z_{r, j}^{1}+z_{1, j}^{1}\right) \\
& \text { FDMA }: \frac{2848 \lambda_{j}^{0}}{a_{1} a_{s}} \tilde{z}_{r, j}^{s}=\frac{2848 \lambda_{j}^{0}}{a_{1} a_{s}} \quad\left(a_{1} z_{r, j}^{s}-a_{1} z_{1, j}^{s}-a_{s} z_{r, j}^{1}+a_{s} z_{1, j}^{1}\right)
\end{aligned}
$$

where one can verify that the two ambiguities are equivalent if $a_{1}=\cdots=a_{s}=2848$, implying that the CDMA model is a special case of the FDMA model. In other words, the FDMA model can be directly transformed to the CDMA model by setting the frequencies of all satellites to the same. This illustrates that we establish a unified framework for CDMA and FDMA PPP-RTK models.

The coefficients of the ambiguities determine the performance of ambiguity resolution. One may question the success rate of FDMA integer ambiguity resolution as the coefficients are so small (approximately $0.1 \mathrm{~mm}$ ). To ensure rigorous ambiguity resolution and improve the success rate, we must transform the estimable FDMA ambiguities in Eq. (55) into integer-estimable ambiguities defined in Teunissen (2019). We analyze the performance of GLONASS ambiguity resolution through (Teunissen, 2019)

$$
\eta_{\text {DF-FDMA }} \approx\left(\frac{2848}{g_{m}}\right)^{\frac{1}{(m-1)}} \eta_{\text {DF-CDMA }}
$$

where $\eta_{\mathrm{DF}-\mathrm{FDMA}}$ denotes the dual-frequency FDMA Ambiguity Dilution Of Precision (ADOP), while $\eta_{\mathrm{DF}-\mathrm{CDMA}}$ denotes the CDMA ADOP taking dual-frequency GPS as an example. $g_{m}$ denotes the greatest common divisor of $\left(a_{1}, \ldots, a_{m}\right)$.

Equation (56) indicates that GLONASS ambiguity resolution depends highly on the number of satellites. Since $g_{m}$ is at most two for seven satellites, the FDMA ADOP is approximately three and a half times larger than its CDMA counterpart, indicating a lower FDMA ambiguity success rate. Fortunately, partial ambiguity resolution can remarkably improve the FDMA ambiguity success rate by resolving per frequency $m-2$ ambiguities instead of $m-1$. In this way, FDMA ambiguity resolution performs as well as CDMA ambiguity resolution (Teunissen, 2019).

While many studies have validated the CDMA PPPRTK (Odijk et al., 2017; Psychas et al., 2021; Teunissen et al., 2010), the FDMA PPP-RTK is a recent progress. For an initial assessment of the FDMA PPP-RTK, refer to Zhang et al. (2021), in which they succeeded in FDMA ambiguity resolution and high-precision GLONASS PPP-RTK positioning using both the ionosphere-float and ionosphere-weighted models.

\section{lonosphere-float vs. ionosphere-weighted vs. ionosphere-fixed}

Different constraints on tropospheric and ionospheric delays yield three models: ionosphere-float, ionosphereweighted, and ionosphere-fixed models. Table 1 summarizes these constraints on atmospheric delays and their estimable forms in the three models based on code-plusphase observations. The ionosphere-float model avoids any external constraints on atmospheric delays, the ionosphere-weighted model imposes a zero-mean weighted 
Table 1 Constraints on atmospheric delays and their estimable forms in the code-plus-phase ionosphere-float, ionosphere-weighted, and ionosphere-fixed models

\begin{tabular}{llll}
\hline Model & Parameter & Constraint & Estimable form \\
\hline lonosphere-float & Troposphere & None & $\tau_{r}$ \\
& lonosphere & None & $\bar{l}_{r}^{s}=l_{r}^{s}-d_{, \mathrm{GF}}^{s}+d_{r, \mathrm{GF}}$ \\
lonosphere-weighted & Troposphere & $m_{1}^{s}=\cdots=m_{n}^{s}$ & $\tilde{\tau}_{r}=\tau_{r}-\tau_{1}$ \\
& lonosphere & $0=l_{r}^{s}-l_{1}^{s}$ & $\tilde{I}_{r}^{s}=l_{r}^{s}-d_{, \mathrm{GF}}^{s}+d_{1, \mathrm{GF}}$ \\
lonosphere-fixed & Troposphere & $m_{1}^{s} \tau_{1}=\cdots=m_{n}^{s} \tau_{n}=\tau^{s}$ & None \\
& lonosphere & $l_{1}^{s}=\cdots=l_{n}^{s}=l^{s}$ & $\bar{l}=l^{s}-d_{, \mathrm{GF}}^{s}+d_{1, \mathrm{GF}}$ \\
& & & \\
\hline
\end{tabular}

constraint on ionospheric delays and considers the tropospheric mapping functions of all stations identical, while the ionosphere-fixed model assumes the atmospheric delays at all stations identical. As a result, three models of full-rank estimate the original tropospheric delay, between-receiver single-differenced tropospheric delay, and "None", respectively. The "None" means that tropospheric delays of the ionosphere-fixed model are inestimable since they are absorbed into satellite clock errors. Regarding ionospheric delays, the ionosphere-float model estimates the ionospheric delay containing $d_{r, \mathrm{GF}}$, while the other two models estimate the ionospheric delay containing $d_{1, \mathrm{GF}}$. This difference brings more convenient ionospheric modeling for the ionosphere-weighted model since the ionospheric delays of all receivers absorb a common receiver bias, while ionospheric modeling for the ionosphere-float model should consider the inconsistent receiver bias datum (Zha et al., 2021). The ionospherefixed model estimates the same ionospheric delays of all receivers and thus can be directly sent to users without modeling.

In addition to atmospheric parameters, Table 2 shows other parameters that are also different in three models. Since the change in tropospheric constraints affects the rank deficiency between tropospheric delays and satellite clock errors, ionosphere-weighted and ionosphere-fixed models estimate the satellite clock errors absorbing tropospheric delays at the first receiver, while the ionosphere-float model estimates the satellite clock errors without tropospheric delays. Due to the linear correlation between the ionospheric delays and receiver biases, ionospheric constraints on the ionosphereweighted and ionosphere-fixed models change the estimable forms of receiver biases. Compared with the ionosphere-float model, the other two models estimate additionally a Between-Receiver Differential Code Bias (BR-DCB) and estimate the receiver phase bias without geometry-free receiver code bias.

Due to the different atmospheric constraints underlying three models, their applications and performances are different. The ionosphere-float model is suitable for large-scale networks where the ionospheric delays are modeled without any constraints, e.g., the global or national networks. The ionosphereweighted model is designed for medium-scale networks, e.g., the network of a province. One may also carry out ionosphere-float PPP-RTK in medium-scale networks. However, it underperforms the ionosphereweighted PPP-RTK with a proper stochastic model of

Table 2 Different estimable parameters in the code-plus-phase ionosphere-float, ionosphere-weighted, and ionosphere-fixed models

\begin{tabular}{|c|c|c|c|}
\hline \multirow[t]{2}{*}{ Parameter } & \multicolumn{3}{|l|}{ Estimable form } \\
\hline & lonosphere-float & lonosphere-weighted & lonosphere-fixed \\
\hline Satellite clock & $\mathrm{d} \tilde{t}^{s}=\mathrm{d} \bar{t}^{s}-\mathrm{d} \bar{t}_{1}$ & $\mathrm{~d} \tilde{\tilde{t}}^{s}=\mathrm{d} \bar{t}^{s}-\mathrm{d} \bar{t}_{1}-m_{1}^{s} \tilde{\tau}_{1}$ & $\mathrm{~d} \bar{t}^{-s}=\mathrm{d} \bar{t}^{s}-\mathrm{d} \bar{t}_{1}-\tau^{s}$ \\
\hline BR-DCB & None & $\tilde{d}_{r, \mathrm{GF}}=d_{r, \mathrm{GF}}-d_{1, \mathrm{GF}}$ & $\tilde{d}_{r, \mathrm{GF}}=d_{r, \mathrm{GF}}-d_{1, \mathrm{GF}}$ \\
\hline Receiver phase bias & $\begin{array}{l}\tilde{\delta}_{r, j}=\delta_{1 r, j}-d_{1 r, \mathrm{~F}} \\
+u_{j} d_{1 r, \mathrm{GF}}+\lambda_{j} z_{1 r, j}^{1}\end{array}$ & $\tilde{\tilde{\delta}}_{r, j}=\delta_{1 r, j}-d_{1 r, \mathbb{F}}+\lambda_{j j} z_{1 r, j}^{1}$ & $\tilde{\tilde{\delta}}_{r, j}=\delta_{1 r j}-d_{1 r, \mathbb{I} F}+\lambda_{j} z_{1 r, j}^{1}$ \\
\hline
\end{tabular}

(.) ${ }_{1 r}$ denotes a between-receiver single-differenced operation 
ionospheric constraints. In small-scale networks, the ionosphere-fixed model works best compared to the other two models.

This work systematically formulates a class of PPPRTK functional models and casts them in a unified theoretical framework, while many studies have elaborated on the practical aspects of these models. For instance, Zhang et al. (2018) applied the ionosphere-fixed model to analyze the characteristics of the receiver phase bias in a small network, Zha et al. (2021) evaluated the performance of ionosphere-weighted PPP-RTK during low and high solar activity days, and Zhang et al. (2019) carried out ionosphere-float PPP-RTK over China.

\section{Code-plus-phase vs. phase-only}

The code-plus-phase and phase-only PPP-RTK models provide network products in different forms. Table 3 shows the estimable forms of network products based on the two models. Since we form the phase-only model by starting with phase observation equations in the full-rank code-plus-phase model, the estimable parameters of the phase-only model are the linear combinations of the estimable parameters of the codeplus-phase model. One can obtain the same phase-only model if starting with the original phase observation equations. This illustrates that the null space of the code-plus-phase model is a subset of the null space of the phase-only model. Notably, satellite clock and ionospheric products $\left(\mathrm{d} \tilde{\hat{t}}^{s}\right.$ and $\left.\hat{l}_{r}^{s}\right)$ based on phase-only observations absorb ambiguities since they are biased with the estimable satellite phase bias of the code-plusphase model $\tilde{\delta}_{\text {,IF }}^{s}$ that contains ambiguities. This indicates that the magnitudes of the satellite clock error and ionospheric delays are unpredictable, which should be considered when coding and broadcasting the products on the network side.

Despite different forms of individual products based on two models, the combined products are the same. See below the estimable forms of the combined products based on two models

$$
\begin{aligned}
& \mathrm{d} \tilde{t}^{s}+\tilde{l}_{1}^{s}+\tilde{\delta}_{, 1}^{s}=\mathrm{d} t^{s}-\mathrm{d} t_{1}-m_{r}^{s} \tau_{1}+l_{1}^{s} \\
& \quad+\delta_{1}^{s}-\delta_{1,1}-\lambda_{1} z_{1,1}^{s}=-\left(\phi_{1,1}^{s}-\rho_{1}^{s}\right) \\
& \tilde{\hat{t}}^{s}+\tilde{\hat{l}}_{1}^{s}=\mathrm{d} t^{s}-\mathrm{d} t_{1}-m_{r}^{s} \tau_{1}+l_{1}^{s} \\
& \quad+\delta_{, 1}^{s}-\delta_{1,1}-\lambda_{1} z_{1,1}^{s}=-\left(\phi_{1,1}^{s}-\rho_{1}^{s}\right)
\end{aligned}
$$

where $\rho_{1}^{s}$ is the satellite-to-receiver geometrical distance. The phase-only model excludes the satellite phase bias since only observations at the third frequency and above require this product.

Equation (57) illustrates that both code-plus-phase and phase-only PPP-RTK models provide users with the same combined products which can also be represented as (pseudo) observations. This illustrates the transformation from SSR to OSR (Khodabandeh \& Teunissen, 2015). Hence, the RTK terminals can also use the PPP-RTK network products, implying that PPP-RTK is compatible with RTK.

Phase-only ambiguity resolution is more difficult than code-plus-phase ambiguity resolution, especially for the ionosphere-float models. Phase-only ionospherefloat PPP-RTK selects the ambiguities at the first frequency as the S-basis to eliminate the rank deficiencies, making the estimable ambiguities changed. Here, we write again the estimable CDMA ambiguities in the code-plus-phase and phase-only ionosphere-float models

$$
\begin{aligned}
& \text { Code-plus-phase : } \lambda_{j} \tilde{z}_{r, j}^{s}=\lambda_{j}\left(z_{r, j}^{s}-z_{1, j}^{s}-z_{r, j}^{1}+z_{1, j}^{1}\right) \\
& \text { Phase-only : } \frac{\lambda_{j}}{w_{1, j}} \hat{\hat{z}}_{r, j}^{s}=\frac{\lambda_{j}}{w_{1, j}}\left(w_{1, j} \tilde{z}_{r, j}^{s}-w_{2, j} \tilde{z}_{r, 1}^{s}\right)
\end{aligned}
$$

where the phase-only ambiguities are related to the simplest integer ratio of wavelength. Taking dual-frequency GPS observations as an example, its estima-

\begin{tabular}{|c|c|c|}
\hline \multirow[t]{2}{*}{ Parameter } & \multicolumn{2}{|l|}{ Estimable form } \\
\hline & Code-plus-phase & Phase-only \\
\hline Tropospheric delay & $\tilde{\tau}_{r}=\tau_{r}-\tau_{1}$ & $\tilde{\tau}_{r}=\tau_{r}-\tau_{1}$ \\
\hline lonospheric delay & $\tilde{l}_{r}^{s}=l_{r}^{s}-d_{, G F}^{s}+d_{1, \mathrm{GF}}$ & $\tilde{\hat{l}}_{r}^{s}=\tilde{l}_{r}^{s}-\tilde{\tilde{\delta}}_{1, \mathrm{GF}}+\tilde{\delta}_{\mathrm{GF}}^{s}$ \\
\hline Satellite clock & $\mathrm{d} \tilde{\tilde{t}}^{s}=\mathrm{d} \bar{t}^{s}-\mathrm{d} \bar{t}_{1}-m_{1}^{s} \tilde{\tau}_{1}$ & $\mathrm{~d} \tilde{\hat{t}}^{s}=\mathrm{d} \tilde{t}^{s}+\tilde{\delta}_{, I F}^{s}$ \\
\hline Satellite code bias & $\tilde{d}_{j}^{s}=\bar{d}_{j}^{s}-\bar{d}_{1, j}(j>2)$ & None \\
\hline Satellite phase bias & $\tilde{\delta}_{j}^{s}=\bar{\delta}_{j}^{s}-\bar{\delta}_{1, j}-\lambda_{j} z_{1, j}^{s}$ & $\hat{\delta}_{j}^{s}=\tilde{\delta}_{j}^{s}-\tilde{\delta}_{, \mathrm{IF}}^{s}-\mu_{j} \tilde{\delta}_{, \mathrm{GF}}^{s}(j>2)$ \\
\hline
\end{tabular}
ble phase-only ambiguities at the second frequency are $\frac{\lambda_{2}}{60}\left(60 \tilde{z}_{r, 2}^{s}-77 \tilde{z}_{r, 1}^{s}\right)$. The wavelength of the estimable phase-only ambiguities is 60 times smaller than that of code-plus-phase ambiguities, leading to more difficulties

Table 3 Network products based on the code-plus-phase and phase-only ionosphere-weighted models 


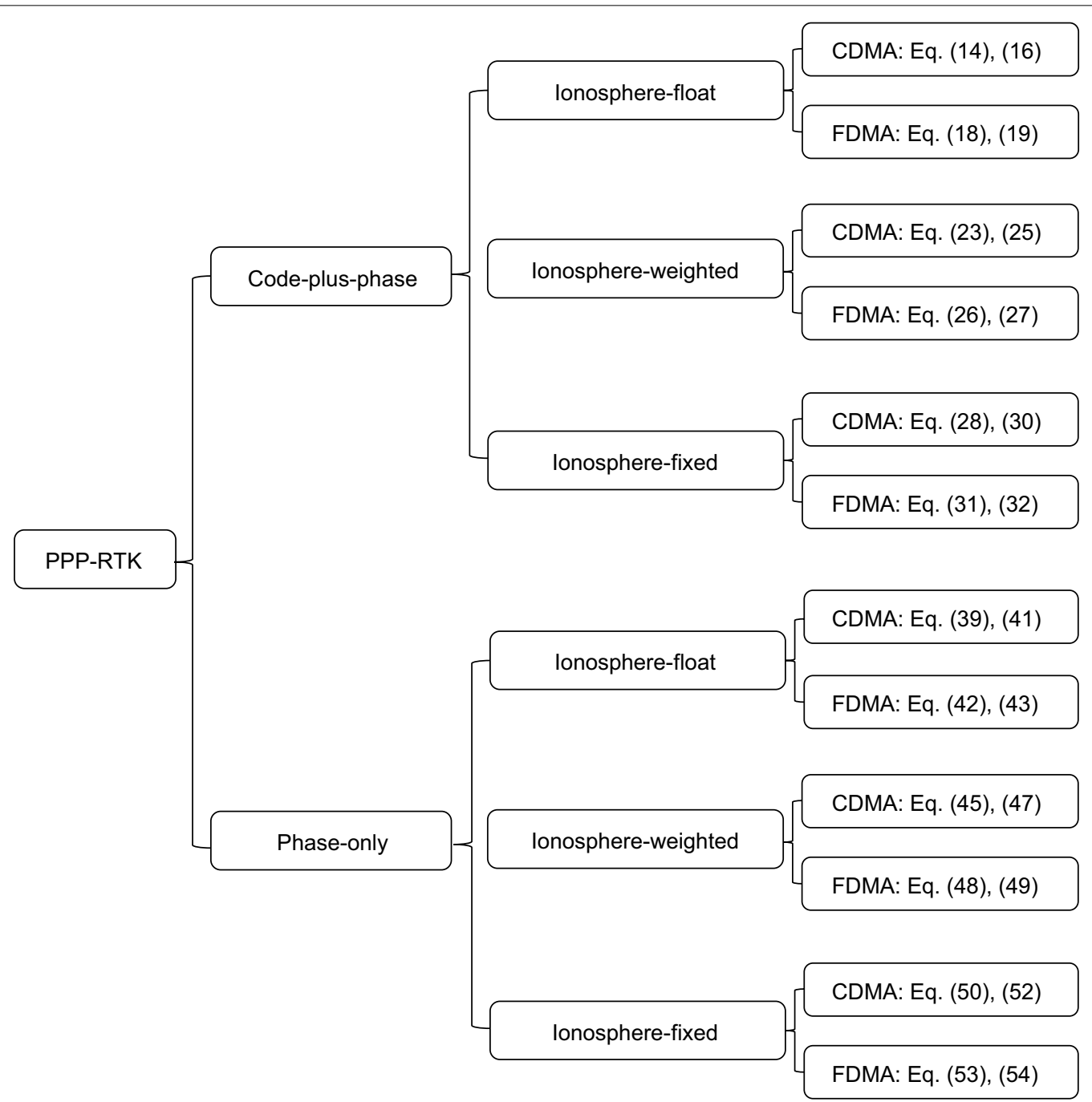

Fig. 1 Framework of undifferenced and uncombined PPP-RTK. Two equations in each textbox on the far right side represent the network and user models

in phase-only ambiguity resolution for the ionospherefloat model. Fortunately, the phase-only ionosphereweighted and ionosphere-fixed models parameterize the same ambiguities as those of the code-plus-phase models. In such cases, the performance of phase-only ambiguity resolution is similar to that of code-plus-phase ambiguity resolution since the contribution of code observations is marginal as its precision is less than that of the phase counterpart by two orders of magnitude.

Although the code-plus-phase model is stronger than its phase-only counterpart, the phase-only model plays an essential role in the case the unmodeled coderelated errors are severe. For instance, in a network of heterogeneous receivers, Zhang et al. (2021) suggested adopting the phase-only model to realize GLONASS
PPP-RTK, thereby avoiding the code IFB. Regarding the CDMA systems, Hou et al. (2022) applied the phase-only model to BDS data and improved the positioning performance compared to the code-plusphase PPP-RTK since the remarkable code multipath degraded the code-plus-phase positioning.

\section{Conclusions}

In this contribution, we present a class of undifferenced and uncombined PPP-RTK functional models in a systematic way. Figure 1 presents these models and shows the framework of undifferenced and uncombined PPP-RTK. We classify the models into the code-plusphase and phase-only divisions. Both divisions contain the ionosphere-float, ionosphere-weighted, and 
ionosphere-fixed models. Each model considers both CDMA and FDMA systems. We conclude the properties of these models below.

Code-plus-phase models are stronger than their phaseonly counterparts, but phase-only models may perform better in the case the unmodeled code-related errors are severe. The exclusion of code observations results in additional rank deficiencies, making more phase-related parameters inestimable. As a result, the estimable parameters of the phase-only model are the combinations of estimable parameters of the code-plus-phase model. The less estimable parameters of the phase-only model indicate the weakness of the model. However, the phase-only models avoid the adverse effects of the unmodeled coderelated errors, such as the code IFB in GLONASS and code multipath in BDS GEO satellites. GLONASS code IFB between different types of receivers can reach several meters, while phase IFB is small enough to be ignored in most practical cases (Wanninger, 2012). Hence, phaseonly GLONASS PPP-RTK fits well for the networks deploying heterogeneous receivers. Many studies have reported that BDS GEO satellites exhibit remarkable code multipath (Montenbruck et al., 2013), implying the potential applications of the phase-only model in such a case.

The ionosphere-weighted model is stronger than the ionosphere-float model, and the ionosphere-fixed model is the strongest. However, this does not mean that the ionosphere-fixed model always performs best. In smallscale networks where atmospheric delays are almost identical at all stations, the ionosphere-fixed model benefits its strength and would perform best. However, in medium-scall networks where one cannot consider all ionospheric delays identical in a deterministic way, the ionosphere-weighted model that imposes weighted constraints on ionospheric delays could perform best. This is because the ionosphere-fixed model is no longer unbiased in medium-scale networks. Concerning large-scale networks, the ionosphere-float model takes the center stage since the weighted constraints on ionospheric delays lose efficiency.

FDMA ambiguity resolution is more difficult than the CDMA ambiguity resolution, but fast FDMA ambiguity resolution is feasible. Due to the effects of IFB, FDMA PPP-RTK parameterizes a specific linear combination of ambiguities, instead of the double-differenced ambiguities in CDMA systems. Taking advantage of the integerestimability theory (Teunissen, 2019), FDMA PPP-RTK can achieve fast partial ambiguity resolution and improve positioning compared to the ambiguity-float solution. The rigorous GLONASS PPP-RTK model contributes to multi-GNSS solutions that provide more accurate, reliable, and continuous positioning services.
In conclusion, all models presented in this contribution play an indispensable role in different scenarios. With the contribution of this work, the GNSS community would enjoy a systematic theoretical framework of undifferenced and uncombined PPP-RTK functional models. We may inevitably miss some materials. For instance, the single-frequency PPP-RTK functional model (Odijk et al., 2012) is not introduced in this contribution, but it can also be cast in the framework shown in Fig. 1. The stochastic and dynamic models are not discussed in this contribution, but they can be flexibly constructed since our functional models are based on undifferenced and uncombined observations and preserve all of the parameters. Some problems remain to be solved. For instance, how to efficiently process the mass data collected by hundreds or even thousands of receivers, which will be addressed in our future work.

\section{Acknowledgements}

Not applicable.

\section{Authors' contributions}

BZ proposed the general idea of this contribution. BZ and PH wrote the manuscript. All authors read and approved the final manuscript.

\section{Funding}

This work was partially funded by the National Natural Science Foundation of China (Grant Nos. 41774042, 42174034), the Key Research and Development Plan of Hubei Province (Grant No. 2020BHB014), and the Scientific Instrument Developing Project of the Chinese Academy of Sciences (Grant No. YJKYYQ20190063). The first author is supported by the CAS Pioneer Hundred Talents Program

\section{Availability of data and materials}

Not applicable.

\section{Declarations}

\section{Competing interests}

The authors declare that they have no competing interests.

\section{Author details}

${ }^{1}$ State Key Laboratory of Geodesy and Earth's Dynamics, Innovation Academy for Precision Measurement Science and Technology, Chinese Academy of Sciences, Wuhan, China. ${ }^{2}$ College of Earth and Planetary Sciences, University of Chinese Academy of Sciences, Beijing, China. ${ }^{3}$ State Key Laboratory of Satellite Navigation System and Equipment Technology, The 54th Research Institute of China Electronics Technology Group Corporation, Shijiazhuang, China.

Received: 26 November 2021 Accepted: 13 January 2022

Published online: 14 February 2022

\section{References}

Collins, P., Bisnath, S., Lahaye, F., \& Héroux, P. (2010). Undifferenced GPS ambiguity resolution using the decoupled clock model and ambiguity datum fixing. Navigation, 57(2), 123-135.

Gao, Y., \& Shen, X. (2002). A new method for carrier-phase-based precise point positioning. Navigation, 49(2), 109-116.

Ge, M., Gendt, G., Ma, R., Shi, C., \& Liu, J. (2008). Resolution of GPS carrier-phase ambiguities in precise point positioning (PPP) with daily observations. Journal of Geodesy, 82(7), 389-399. 
Geng, J., Shi, C., Ge, M., Dodson, A. H., Lou, Y., Zhao, Q., \& Liu, J. (2012). Improving the estimation of fractional-cycle biases for ambiguity resolution in precise point positioning. Journal of Geodesy, 86(8), 579-589.

Gu, S., Shi, C., Lou, Y., \& Liu, J. (2015). Ionospheric effects in uncalibrated phase delay estimation and ambiguity-fixed PPP based on raw observable model. Journal of Geodesy, 89(5), 447-457.

Hou, P., Zhang, B., Yasyukevich, Y. V., Liu, T., Zha, J. (2022). Multi-frequency phase-only PPP-RTK model applied to BeiDou data. GPS Solutions (under review).

Khodabandeh, A. (2021). Single-station PPP-RTK: Correction latency and ambiguity resolution performance. Jornal of Geodesy, 95(4), 1-24. https:// doi.org/10.1007/s00190-021-01490-z

Khodabandeh, A., \& Teunissen, P. (2015). An analytical study of PPP-RTK corrections: Precision, correlation and user-impact. Journal of Geodesy, 89(11), 1109-1132

Khodabandeh, A., \& Teunissen, P. J. G. (2016). PPP-RTK and inter-system biases: The ISB look-up table as a means to support multi-system PPP-RTK. Journal of Geodesy, 90(9), 837-851.

Kouba, J., \& Héroux, P. (2001). Precise point positioning using IGS orbit and clock products. GPS Solutions, 5(2), 12-28.

Lannes, A., \& Prieur, J.-L. (2013). Calibration of the clock-phase biases of GNSS networks: The closure-ambiguity approach. Journal of Geodesy, 87(8), 709-731.

Laurichesse, D., Mercier, F., Berthias, J. P., Broca, P., \& Cerri, L. (2009). Integer ambiguity resolution on undifferenced GPS phase measurements and its application to PPP and satellite precise orbit determination. Navigation, 56(2), 135-149.

Leick, A., Rapoport, L., \& Tatarnikov, D. (2015). GPS satellite surveying. Wiley.

Li, X., \& Ge, Z. M. (2011). Regional reference network augmented precise point positioning for instantaneous ambiguity resolution. Journal of Geodesy, 85(3), 151-158

Liu, T., Yuan, Y., Zhang, B., Wang, N., Tan, B., \& Chen, Y. (2017). Multi-GNSS precise point positioning (MGPPP) using raw observations. Journal of Geodesy, 91(3), 253-268.

Ma, H., Zhao, Q., Verhagen, S., Psychas, D., \& Xianglin, L. (2020). Assessing the performance of multi-GNSS PPP-RTK in the local area. Remote Sensing, 12(20), 3343.

Montenbruck, O., Hauschild, A., Steigenberger, P., Hugentobler, U., Teunissen, P., \& Nakamura, S. (2013). Initial assessment of the COMPASS/BeiDou-2 regional navigation satellite system. GPS Solutions, 17(2), 211-222.

Naciri, N., \& Bisnath, S. (2021). An uncombined triple-frequency user implementation of the decoupled clock model for PPP-AR. Journal of Geodesy, 95(5), 60 .

Odijk, D. (2002). Fast precise GPS positioning in the presence of ionospheric delays. In Publications on geodesy, 52, Netherlands Geodetic Commission, Delft, The Netherlands.

Odijk, D., Khodabandeh, A., Nadarajah, N., Choudhury, M., \& Zhang, B. (2017). PPP-RTK by means of S-system theory: Australian network and user demonstration. Journal of Spatial Science, 62(1), 3-27.

Odijk, D., Teunissen, P. J. G., \& Zhang, B. (2012). Single-frequency integer ambiguity resolution enabled GPS precise point positioning. Journal of Surveying Engineering, 138(4), 193-202.

Odijk, D., Zhang, B., Khodabandeh, A., Odolinski, R., \&Teunissen, P. J. G. (2016) On the estimability of parameters in undifferenced, uncombined GNSS network and PPP-RTK user models by means of S-system theory. Journal of Geodesy, 90(1), 15-44.

Psychas, D., Teunissen, P. J. G., \& Verhagen, S. (2021). A multi-frequency Galileo PPP-RTK convergence analysis with an emphasis on the role of frequency spacing. Remote Sensing, 13(16), 3077.

Schönemann, E., Becker, M., \& Springer, T. (2011). A new approach for GNSS analysis in a multi-GNSS and multi-signal environment. Journal of Geodetic Science, 1(3), 204-214.

Teunissen, P. J. G. (1985). Generalized inverses, adjustment, the datum problem and S-transformations. In E. W. Grafarend \& F. Sanso (Eds.), Optimization of Geodetic Networks. Springer.

Teunissen, P. J. G. (2019). A new GLONASS FDMA model. GPS Solutions, 23(4)، 1-19. https://doi.org/10.1007/s10291-019-0889-0

Teunissen, P., \& Khodabandeh, A. (2015). Review and principles of PPP-RTK methods. Journal of Geodesy, 89(3), 217-240.

Teunissen, P. J. G., \& Montenbruck, O. (2017). Springer handbook of global navigation satellite systems. Springer.
Teunissen, P. J. G., Odijk, D., \& Zhang, B. (2010). PPP-RTK: Results of CORS network-based PPP with integer ambiguity resolution. Journal of Aeronautics, Astronautics and Aviation, Series A, 42(4), 223-230.

Wang, K., Khodabandeh, A., \& Teunissen, P. (2017). A study on predicting network corrections in PPP-RTK processing. Advances in Space Research, 60(7), 1463-1477

Wanninger, L. (2012). Carrier-phase inter-frequency biases of GLONASS receivers. Journal of Geodesy, 86(2), 139-148.

Wübbena, G., Schmitz, M., \& Bagge, A. (2005). PPP-RTK: precise point positioning using state-space representation in RTK networks. In: Proceedings of the ION GNSS 2005, The Institute of Navigation. Long Beach, CA, September 13-16

Wübbena, G., Bagge, A., \& Schmitz, M. (2001). Network-based techniques for RTK applications. Proceedings of GPS Symposium, 2001, 14-16.

Zha, J., Zhang, B., Liu, T., \& Hou, P. (2021). lonosphere-weighted undifferenced and uncombined PPP-RTK: Theoretical models and experimental results. GPS Solutions, 25(4), 1-12.

Zhang, B., Chen, Y., \& Yuan, Y. (2019). PPP-RTK based on undifferenced and uncombined observations: Theoretical and practical aspects. Journal of Geodesy, 93(7), 1011-1024.

Zhang, B., Hou, P., Zha, J., \& Liu, T. (2021). Integer-estimable FDMA model as an enabler of GLONASS PPP-RTK. Journal of Geodesy, 95(8), 1-21. https://doi. org/10.1007/s00190-021-01546-0

Zhang, B., Liu, T., \& Yuan, Y. (2018). GPS receiver phase biases estimable in PPP_ RTK networks: Dynamic characterization and impact analysis. Journal of Geodesy, 92(6), 659-674.

Zhang, B., Teunissen, P. J. G., \& Odijk, D. (2011). A novel un-differenced PPP-RTK concept. Journal of Navigation, 64(S1), S180-S191.

Zumberge, J., Heflin, M., Jefferson, D., Watkins, M., \& Webb, F. (1997). Precise point positioning for the efficient and robust analysis of GPS data from large networks. Journal of Geophysical Research: Solid Earth, 102(B3), 5005-5017.

\section{Publisher's Note}

Springer Nature remains neutral with regard to jurisdictional claims in published maps and institutional affiliations. 